\title{
Decrease of Serum IGF-1 Level is not Associated With Obstructive Sleep Apnea in Fibromyalgia Patients
}

\author{
Emrullah HAYTA, ${ }^{1}$ Ayşe Altun OKŞAŞOĞLU² \\ ${ }^{1}$ Department of Physical Medicine and Rehabilitation, Medical Faculty of Cumhuriyet University, Sivas, Turkey \\ ${ }^{2}$ Department of Physical Medicine and Rehabilitation, Sivas Numune Hospital, Sivas, Turkey
}

\begin{abstract}
Objectives: This study aims to investigate the relationship of serum insulin-like growth factor 1 (IGF-1) level with obstructive sleep apnea, the Pittsburgh sleep quality index (PSQI), age, body mass index, and fibromyalgia impact questionnaire (FIQ) in fibromyalgia syndrome (FMS) patients.

Patients and methods: A total of 105 female patients (mean age $41.8 \pm 9.0$ years; range 26 to 55 years) with fibromyalgia (FMS group) who were diagnosed according to 2010 American College of Rheumatology criteria, and 51 female patients (mean age $39.9 \pm 10.8$ years; range 24 to 54 years) with mechanical low back pain (MLBP) (control group) were included in the study. Age, smoking, educational status, tender point number, body mass index, duration of disease, FIQ, PSQI, and polysomnographic assessment of both FMS and control groups were recorded.

Results: Sleep disorder was detected in 88 patients in FMS group and 15 patients in control group $(\mathrm{p}<0.05)$. The PSQI score was higher in the FMS group compared to the control group ( $9.9 \pm 4.6$ vs $5.7 \pm 3.5$ ). The FIQ score was higher in the FMS group compared to the control group (53.4 \pm 17.4 vs $26.4 \pm 13.9 ; \mathrm{p}<0.05)$. The serum IGF-1 level of FMS group was significantly lower than that of the control group $(140.6 \pm 49.5 \mathrm{ng} / \mathrm{mL} \mathrm{vs} 177.2 \pm 58.5 \mathrm{ng} / \mathrm{mL}$; $\mathrm{p}<0.05)$. In the FMS group, an examination of the correlation between serum IGF-1 level with the age, body mass index, obstructive sleep apnea syndrome, FIQ, and PSQI revealed a negative correlation between serum IGF-1 with the age and PSQI. The obstructive sleep apnea syndrome ratios of study groups were comparable with regard to the frequencies of mild, moderate, and severe obstructive sleep apnea syndrome.

Conclusion: In FMS patients, serum IGF-1 levels may decrease due to age and PSQI; however, this may not be related to the severity of obstructive sleep apnea.

Keywords: Fibromyalgia; insulin-like growth factor 1; obstructive sleep apnea; polysomnography.
\end{abstract}

Fibromyalgia syndrome (FMS) is a chronic, non-articular and non-inflammatory pain syndrome characterized by common musculoskeletal system pain and painful sensitive spots in certain regions of the body whose etiology and pathophysiological mechanisms are not fully discovered. ${ }^{1}$ It is believed that there are several mechanisms contributing to the development of FMS. Evidence has been found on some biochemical, neurohormonal, central nervous system, immunological, psychological, and environmental factors which are thought to play role in etiology of FMS. ${ }^{2-4}$ In several studies which researched the etiopathogenesis of FMS, it is argued that it is a neuroendocrine disorder. ${ }^{5}$ It is suggested that abnormalities in hormone levels or the deficiencies in hormonal responses to various stimuli might be effective in occurrence of FMS. ${ }^{6,7}$ The major neuroendocrine disorder defined in patients with FMS is the decline in the release of growth hormone $(\mathrm{GH})$ and insulin-like growth factor 1 (IGF-1). ${ }^{8,9}$ Several clinical findings in FMS such as tiredness, low exercising capacity and cold intolerance can be explained by $\mathrm{GH}$ deficiency. It is also argued that $\mathrm{GH}$ further declines as FMS progresses. ${ }^{10}$ Of daily $\mathrm{GH}, 70 \%$ release takes place at night during non-rapid eye movement (non-REM) phase 3 (especially, during phases 3 and 4 of non$\mathrm{REM}$ sleep). Therefore, sleep disorder is one of the major factors that affect $\mathrm{GH}$ release. ${ }^{11,12}$ 
Sleeping disorders such as insomnia, waking up early, unrefreshing sleep, and poor-quality sleep are frequently experienced by patients with FMS. Waking up unrefreshed in the morning is one of the symptoms that are included in the symptom severity score among 2010 American College of Rheumatology criteria. Although FMS sleep disorder occurs at a frequency of 70 to $90 \%$, it is rarely associated with other FMS symptoms. ${ }^{13-15}$ Initial studies to investigate sleep disorders in patients with FMS experience were launched three decades ago. These studies found that patients with FMS had abnormality in delta wave in the deepest phase of sleep, as well as electroencephalogram (EEG) pattern of non-REM sleep which is disrupted by alpha waves of $10-12$ cycles/second. ${ }^{16,17}$ In addition to studies conducted through EEG in FMS, there have been recent polysomnography (PSG) studies examining the characteristics of sleep. These studies found that the frequency of obstructive sleep apnea syndrome (OSAS) among patients with FMS tended to increase. Nevertheless, there are clinical studies in the literature reporting that the increase of OSAS in FMS is coincidental and unrelated with FMS symptoms. ${ }^{18,19}$

Different studies are available in the literature measuring IGF-1 level and examining sleep disorder. However, to our knowledge, there is no study which investigates sleep disorder in FMS through PSG, and how IGF-1 release is consequently affected in patients with sleep disorders, as well as studying the relationship between the two. Therefore, in this study, we aimed to investigate the relationship of serum IGF-1 level with obstructive sleep apnea, the Pittsburgh sleep quality index (PSQI), age, body mass index (BMI), and fibromyalgia impact questionnaire (FIQ) in FMS patients.

\section{PATIENTS AND METHODS}

A total of 105 female patients (mean age $41.8 \pm 9.0$ years; range 26 to 55 years) who clinically met American College of Rheumatology's FMS diagnosis criteria ${ }^{16,20}$ (FMS group), as well as 51 female patients (mean age $39.9 \pm 10.8$ years; range 24 to 54 years) with mechanical low back pain (MLBP) (control group) were enrolled in the study which was conducted at Cumhuriyet
University Faculty of Medicine, Department of Physical Medicine And Rehabilitation, Sivas, Turkey, between October 2011 and December 2014. Attention was paid to include patients with FMS who had not reached menopause, who had the disease for at least one year, and who did not have secondary FMS. Excluded among FMS patients and MLBP patients were those who had been using thyroid hormone for the last three months, who used oral contraceptive pills, received a previous treatment with $\mathrm{GH}$, pregnant or puerperant patients, those who had intracranial space occupying lesion, and any relevant endocrine disorder including diabetes mellitus, and those with systemic disease (thyroid function disorder, vitamin D deficiency, diabetes mellitus, liver and renal dysfunction, neuropsychiatric disease, morbid obesity, and other systemic or joint inflammatory rheumatic conditions, previous or current malignancies). Age, smoking and educational status, tender point count, BMI, duration of disease, FIQ, PSQI, and polysomnographic assessment of FMS and control groups were recorded. The study protocol was approved by the Faculty Ethics Committee, with number 2010-06/30. A written informed consent was obtained from each participant. The study was conducted in accordance with the principles of the Declaration of Helsinki.

Pittsburgh sleep quality index is a questionnaire assessing subjective sleep quality under seven components, namely sleep quality, sleep latency, sleep duration, habitual sleep efficiency, sleep disturbances, use of sleeping medications, and daytime dysfunction using 19 items. ${ }^{21}$ PSQI was filled in by the same physician who interviewed the patients personally. The questions are rated from 0 to 3 . Seven components are first internally assessed. Afterwards, the scores of these components are added. A total score of 5 or greater is indicative of poor sleep quality. We recorded scores of all subjects. The global score obtained ranges from 0 to 21 , and high scores indicate poor sleep quality and a high level of sleep disorder. A global score of 5 or greater indicates that sleep quality is clinically poor on a significant level.

The $\mathrm{FIQ}^{21}$ is comprised of 10 items, the first of which includes 11 subcategories investigating the ability to conduct large muscle tasks. The rating of each question was conducted on a 
4-point (0 to 3) Likert-type scale. In items 2 and 3, the patients were asked to report about the number of days in which they are fed up with conducting their normal daily tasks and they failed to perform these tasks due to FMS symptoms. Items from 4 to 10 were designed as horizontal linear scales having 10 increments through which work difficulty, pain, fatigue, morning tiredness, stiffness, anxiety, and depression were rated. When the rating was concluded, the emerging scores were normalized. Any of the items can possibly have maximum score of 10 , and the total score can be maximum 100. The higher scores in the questionnaire are, the greater the functional impairment is. In most clinical trials on FMS, the FIQ is one of the tools chiefly recommended to measure the primary efficacy end point.

Full-night PSG was performed in FMS and control groups by a computerized system (SomnoStar alpha; SensorMedics, Anaheim, CA, USA). Polysomnography is a test based on synchronized and continuous recording for certain period, usually nightlong, of neurophysiological, cardiac, respiratory, physiological, and other physical parameters during sleep. In the standard PSG record conducted, there are parameters from EEG, submental and tibia electromyogram, electrooculogram (2 channels) and electrocardiogram, nasal airflow sensor, chest and abdomen movements sensor, body position sensor, tracheal microphone, and pulse oximeter. Apnea hypopnea index (AHI) is the total number of apneas and hypopneas during the sleep divided by sleeping duration as hours. According to this index, the happenings are classified as simple snoring $(\mathrm{AHI}<5)$, mild OSAS (AHI=5-15), moderate OSAS $(\mathrm{AHI}=16-30)$, and severe OSAS (AHI>30). ${ }^{22}$

Blood samples were collected at 8 am in order to determine IGF-1 levels of the FMS and control groups. The level of serum IGF-1 was measured by the immunoradiometric assay method using a commercial kit (Immunotech, A Beckman Coulter Company, France). Berthold LB2111 Gamma Counter was used for the measurement. The measurement range was $2-1200 \mathrm{ng} / \mathrm{mL}$. The intermediate precision was $6.8 \%$ using a coefficient of variation.

\section{Statistical analysis}

Clinical data of the study groups were presented as mean \pm standard deviation or percentage. For the analyses of the clinical data, IBM SPSS Statistics for Windows, Version 22.0 (IBM Corp., Armonk, NY, USA) was used. In the assessment of normality, Kolmogorov-Smirnov test was used. Independent sample $\mathrm{t}$ test was used when the parametric test parameters met the assumptions; however, when the parametric test parameters did not meet the assumptions, Mann-Whitney $\mathrm{U}$ test was used. Chi-square test was used for the comparison of nominal data. Spearman's correlation was used to investigate the correlation of serum IGF-1 level with other clinical variables. In multiple linear regression analysis, the PSQI, age, BMI, FIQ, and OSAS parameters entered into the model. A $p$ value of less than 0.05 was accepted as significant.

\section{RESULTS}

Table 1 presents the demographic and clinical data of study groups. The age, educational status, smoking, and BMI of groups were similar ( $p>0.05)$. Sleep disorder was detected in 88 patients in the FMS group and 15 patients in the control group $(\mathrm{p}<0.05)$. The PSQI score was higher in the FMS group than the control group $(9.9 \pm 4.6$ vs $5.7 \pm 3.5)$. The FIQ score was higher in the FMS group than the control group $(53.4 \pm 17.4$ vs $26.4 \pm 13.9 ; p<0.05)$. A comparison of the IGF-1 serum levels of both groups showed that the level was lower in the FMS group than the control group $(140.6 \pm 49.5 \mathrm{ng} / \mathrm{mL}$ vs $177.2 \pm 58.5 \mathrm{ng} / \mathrm{mL}$, respectively; $\mathrm{p}<0.05$ ).

After Spearman's correlation, the degree of correlation with the serum IGF-1 level with the PSQI, age, BMI, FIQ, and OSAS parameters were assessed and later with stepwise linear regression analysis, we found that there was a negative correlation between the serum IGF-1 level with the age and PSQI parameters in the FMS group $(p<0.05)$. The serum IGF-1 level did not present any meaningful correlation with other parameters ( $p>0.05)$. The $\mathrm{R}$ square value and adjusted $\mathrm{R}$ square value of the model were calculated to be 0.297 and 0.283 , respectively (Table 2 ).

A comparison between FMS and control groups in terms of OSAS frequency revealed that 15, seven, and four patients in the FMS group had mild, moderate, and severe OSAS, respectively; whereas eight, two, and three 
Table 1. Demographic and clinical data of patients with fibromyalgia syndrome and mechanical low back pain

\begin{tabular}{|c|c|c|c|c|c|c|}
\hline & \multicolumn{3}{|c|}{$\begin{array}{c}\text { Fibromyalgia } \\
\text { syndrome }(n=105)\end{array}$} & \multicolumn{3}{|c|}{$\begin{array}{l}\text { Mechanical low } \\
\text { back pain }(n=51)\end{array}$} \\
\hline & $\mathrm{n}$ & $\%$ & Mean \pm SD & $\mathrm{n}$ & $\%$ & Mean \pm SD \\
\hline Age (year) & & & $41.8 \pm 9.0$ & & & $39.9 \pm 10.8$ \\
\hline \multicolumn{7}{|l|}{ Education status } \\
\hline Up to high school & 89 & 84.7 & & 41 & 80.3 & \\
\hline University & 16 & 15.2 & & 10 & 19.7 & \\
\hline \multicolumn{7}{|l|}{ Smoking } \\
\hline Yes & 16 & 15.2 & & 6 & 11.7 & \\
\hline No & 95 & 84.8 & & 89 & 88.3 & \\
\hline \multicolumn{7}{|l|}{ Use of drug } \\
\hline Antidepressant & 4 & 3.8 & & 1 & 2.0 & \\
\hline Antiepileptic & 11 & 10.5 & & 4 & 7.8 & \\
\hline No drug use & 90 & 85.7 & & 47 & 78.4 & \\
\hline Body mass index $\left(\mathrm{m}^{2} / \mathrm{kg}\right)$ & & & $28.7 \pm 3.1$ & & & $28.2 \pm 4.4$ \\
\hline Duration of fibromyalgia syndrome (year) & & & $3.7 \pm 3.1$ & & & \\
\hline Number of tender point & & & $12.8 \pm 3.2$ & & & \\
\hline Sleep disorder & 88 & $83.8 \mathrm{a}$ & & 15 & 29.4 & \\
\hline Pittsburgh sleep quality index & & & $9.9 \pm 4.6 b$ & & & $5.7 \pm 3.5$ \\
\hline Fibromyalgia impact questionnaire score & & & $53.4 \pm 17.4 c$ & & & $26.4 \pm 13.9$ \\
\hline Insulin-like growth factor 1 (ng/mL) & & & $140.6 \pm 49.5 \mathrm{~d}$ & & & $177.2 \pm 58.5$ \\
\hline
\end{tabular}

patients in control group had mild, moderate, and severe OSAS, respectively (Table 3).

\section{DISCUSSION}

Low IGF-1 level has been consistently shown in patients with FMS in several studies in the related literature. ${ }^{[12]}$ However, in those studies, the factors resulting in the decreased levels of IGF-1 have not been investigated in detail. Therefore, the present study investigated the factors affecting the OSAS frequency and IGF-1 level in FMS. We have clearly demonstrated that OSAS frequency in patients with FMS does not increase compared to control subjects with low back pain, and FMS patients' IGF-1 level was lower than that of control subjects. In the FMS patients, in the serum level of IGF-1, although the increase of age and PSQI causes a decrease, severity of OSAS did not present any meaningful change.

Although sleep disorder in FMS occurs frequently at a rate of 70 to $90 \%$, it is rarely associated with other symptoms. Waking up feeling unrefreshed is one of the other two core symptoms included in the symptom severity scale in new 2010 American College of Rheumatology criteria for FMS. ${ }^{24}$ In our study, these conditions were found in $88 \%$ of the patients, and this was in compliance with the literature. Several researchers conducted PSG in order to explore physiological foundations of poor sleep quality in patients with fibromyalgia experience. ${ }^{25,26}$ In their study, Drewes et al. ${ }^{27}$ discovered various changes

Table 2. Multiple linear regression analysis for the serum insulin-like growth factor 1 level in patients with fibromyalgia syndrome

\begin{tabular}{|c|c|c|c|c|c|}
\hline \multirow[b]{3}{*}{ Independent variables } & \multicolumn{4}{|c|}{ Dependent variable (serum IGF-1 level) } & \multirow[b]{3}{*}{$p$} \\
\hline & \multicolumn{2}{|c|}{ Spearman correlation } & \multicolumn{2}{|c|}{ Multiple regression } & \\
\hline & $\mathrm{r}$ & $p$ & $\beta$ & $\mathrm{t}$ & \\
\hline Age & -0.279 & 0.001 & -1.188 & -2.546 & 0.012 \\
\hline Body mass index & -0.212 & 0.001 & -0.1 & -1.105 & 0.272 \\
\hline Pittsburgh sleep quality index & -0.245 & 0.001 & -5.104 & -5.718 & 0.001 \\
\hline Fibromyalgia impact questionnaire & -0.376 & 0.001 & 0.05 & 0.476 & 0.635 \\
\hline Obstructive sleep apnea syndrome & -0.282 & 0.001 & -0.144 & -1.518 & 0.312 \\
\hline
\end{tabular}




\begin{tabular}{|c|c|c|c|c|c|c|c|c|}
\hline \multirow[b]{3}{*}{ Groups } & \multicolumn{8}{|c|}{ Obstructive sleep apnea syndrome } \\
\hline & \multicolumn{2}{|c|}{ No } & \multicolumn{2}{|c|}{ Mild } & \multicolumn{2}{|c|}{ Moderate } & \multicolumn{2}{|c|}{ Severe } \\
\hline & $\mathrm{n}$ & $\%$ & $\mathrm{n}$ & $\%$ & $\mathrm{n}$ & $\%$ & $\mathrm{n}$ & $\%$ \\
\hline Fibromyalgia syndrome & 79 & 75.2 & 15 & 14.3 & 7 & 6.7 & 4 & 3.8 \\
\hline Low back pain & 38 & 74.5 & 8 & 15.7 & 2 & 3.9 & 3 & 5.9 \\
\hline
\end{tabular}

such as disrupted sleep pattern along with delayed occurrence of EEG sleep, while Pivik et al. ${ }^{28}$ found significant alpha (7-12 Hz) EEG non-REM sleep anomaly which is commonly termed in slow wave and REM sleep and non-REM sleep physiology as alpha intrusion or EEG alpha sleep disorder. ${ }^{29}$ Various changes in EEG sleep are noted, such as increased superficial sleep and decreased deep sleep periods, as characteristic PSG findings in patients with OSAS as well as in FMS. Smith et al. ${ }^{31}$ demonstrated in their study that sleep disorder, waking up unrefreshed and PSG changes, which occur in FMS, might also occur in patients with OSAS.

To the best of our knowledge, only a few studies in the literature have investigated the OSAS frequency in patients with FMS. In their study, May et al. ${ }^{32}$ found OSAS frequency in female patients with FMS as insignificant, while they found this frequency as significant in male patients with FMS, which led them to conclude that further research must be performed on male patients in terms of OSAS risk. Moldofsky et al. ${ }^{33}$ found moderate to severe obstructive sleep apnea disorder frequency to be $15.3 \%$. In our study, we found OSAS frequency in female patients to be $10.5 \%$. Although our study yielded similar results to those of Moldofsky et al., ${ }^{33}$ the difference could be attributed to the fact that the patients in our study were younger, had lower BMI, and were only females.

Since sleep disorder and OSAS affect non-REM duration, they might cause lower GH release in patients with FMS. ${ }^{35-37}$ Several studies demonstrated low level of IGF-1 in patients with FMS. This might be explained in relation to evidence from 24-hour $\mathrm{GH}$ profiles in patients with fibromyalgia suggesting that this might be the result of the decrease in $\mathrm{GH}$ secretion. ${ }^{28}$ Besides the decrease in mean $\mathrm{GH}$ secretion, $\mathrm{GH}$ pulse amplitude and area under the $\mathrm{GH}$ pulse curve in patients with fibromyalgia also showed a tendency to decrease. It was argued that the most significant parameter that might affect this low level is failing to enter non-REM sleep, and the decrease may be due to hypothalamic-pituitary-adrenal axis disorder. ${ }^{35-37}$ About $80 \%$ of FMS patients has sleep disturbances which suggests that low $\mathrm{GH}$ secretion may also be due to the incapacity to reach stage 3 and REM sleep. In conformance with other studies in the literature, in our study, we found that IGF-1 was low in patients with FMS, and age and sleep disorder affected IGF-1 level, while there was no relationship between low IGF-1 level and OSAS in patients with FMS. This may be related to the small sample size in our study. Although considerable advances have been made in our understanding of the relative contributions of altered $\mathrm{GH}$ and IGF-1 secretion, further studies are required for the explanation of molecular mechanisms of IGF-1 decrease and FMS-mediated OSAS.

The strength of our study is that, to our knowledge, it is the first study investigating how sleep disorder and OSAS in patients with FMS affect the release of IGF-1. In order to assure consistency in PSG assessment, the study was conducted in a single center. Staying at the hospital overnight for PSG assessment is something which some patients find difficult to consent. Thus, the limitation of this study is its small-numbered sample size.

In conclusion, overall, the OSAS frequency of FMS patients does not change compared to MLBP patients. Serum IGF-1 levels of FMS patients may decrease according to age and PSQI parameters but not with the severity of OSAS. Nevertheless, further studies are required to assess the interaction of obstructive sleep apnea and serum IGF-1 levels in FMS, according to severity of the disorder. 


\section{Declaration of conflicting interests}

The authors declared no conflicts of interest with respect to the authorship and/or publication of this article.

\section{Funding}

The authors received no financial support for the research and/or authorship of this article.

\section{REFERENCES}

1. Wolfe F, Ross K, Anderson J, Russell IJ, Hebert L. The prevalence and characteristics of fibromyalgia in the general population. Arthritis Rheum 1995;38:19-28.

2. Boomershine CS. Fibromyalgia: the prototypical central sensitivity syndrome. Curr Rheumatol Rev 2015;11:131-45.

3. Bennett RM. Fibromyalgia and the disability dilemma. A new era in understanding a complex, multidimensional pain syndrome. Arthritis Rheum 1996;39:1627-34.

4. Okifuji A, Hare BD. Management of fibromyalgia syndrome: review of evidence. Pain Ther 2013;2:87-104.

5. Yunus MB. Editorial review: an update on central sensitivity syndromes and the issues of nosology and psychobiology. Curr Rheumatol Rev 2015;11:70-85.

6. Wolfe F, Russell IJ, Vipraio G, Ross K, Anderson J. Serotonin levels, pain threshold, and fibromyalgia symptoms in the general population. J Rheumatol 1997;24:555-9.

7. Neeck G. Neuroendocrine and hormonal perturbations and relations to the serotonergic system in fibromyalgia patients. Scand J Rheumatol Suppl 2000;113:8-12.

8. Cuatrecasas G, Gonzalez MJ, Alegre C, Sesmilo G, Fernandez-Solà J, Casanueva FF, et al. High prevalence of growth hormone deficiency in severe fibromyalgia syndromes. J Clin Endocrinol Metab 2010;95:4331-7.

9. Yuen KC, Bennett RM, Hryciw CA, Cook MB, Rhoads SA, Cook DM. Is further evaluation for growth hormone $(\mathrm{GH})$ deficiency necessary in fibromyalgia patients with low serum insulin-like growth factor (IGF)-I levels? Growth Horm IGF Res 2007; 17:82-8.

10. Jones KD, Deodhar P, Lorentzen A, Bennett RM, Deodhar AA. Growth hormone perturbations in fibromyalgia: a review. Semin Arthritis Rheum 2007;36:357-79.

11. Dessein PH, Shipton EA, Joffe BI, Hadebe DP, Stanwix AE, Van der Merwe BA. Hyposecretion of adrenal androgens and the relation of serum adrenal steroids, serotonin and insulin-like growth factor-1 to clinical features in women with fibromyalgia. Pain 1999;83:313-9.

12. Van Cauter E, Plat L, Copinschi G. Interrelations between sleep and the somatotropic axis. Sleep 1998;21:553-66.
13. Burns JW, Crofford LJ, Chervin RD. Sleep stage dynamics in fibromyalgia patients and controls. Sleep Med 2008;9:689-96.

14. Wolfe F, Clauw DJ, Fitzcharles MA, Goldenberg DL, Katz RS, Mease P, et al. The American College of Rheumatology preliminary diagnostic criteria for fibromyalgia and measurement of symptom severity. Arthritis Care Res (Hoboken) 2010;62:600-10.

15. Bradley LA, Alarcon GS. Fibromyalgia. In: Koopman WJ, editor. Arthritis and Allied Conditions. Philadelphia: Lippincott Williams \& Wilkins; 2001. p. 1811-44.

16. Moldofsky H, Scarisbrick P. Induction of neurasthenic musculoskeletal pain syndrome by selective sleep stage deprivation. Psychosom Med 1976;38:35-44.

17. Spaeth M, Rizzi M, Sarzi-Puttini P. Fibromyalgia and sleep. Best Pract Res Clin Rheumatol 2011;25:227-39.

18. Clauw DJ. Fibromyalgia: an overview. Am J Med 2009;122:3-13.

19. Sepici V, Tosun A, Köktürk O. Obstructive sleep apnea syndrome as an uncommon cause of fibromyalgia: a case report. Rheumatol Int 2007;28:69-71.

20. Wolfe F, Smythe HA, Yunus MB, Bennett RM, Bombardier C, Goldenberg DL, et al. The American College of Rheumatology 1990 Criteria for the Classification of Fibromyalgia. Report of the Multicenter Criteria Committee. Arthritis Rheum 1990;33:160-72.

21. Burckhardt CS, Clark SR, Bennett RM. The fibromyalgia impact questionnaire: development and validation. J Rheumatol 1991;18:728-33.

22. Epstein LJ, Kristo D, Strollo PJ Jr, Friedman N, Malhotra A, Patil SP, et al. Clinical guideline for the evaluation, management and long-term care of obstructive sleep apnea in adults. J Clin Sleep Med 2009;5:263-76.

23. Wolfe F, Clauw DJ, Fitzcharles MA, Goldenberg DL, Häuser W, Katz RS, et al. Fibromyalgia criteria and severity scales for clinical and epidemiological studies: a modification of the ACR Preliminary Diagnostic Criteria for Fibromyalgia. J Rheumatol 2011;38:1113-22.

24. Diaz-Piedra C, Di Stasi LL, Baldwin CM, Buela-Casal G, Catena A. Sleep disturbances of adult women suffering from fibromyalgia: a systematic review of observational studies. Sleep Med Rev 2015;21:86-99.

25. Spaeth M, Rizzi M, Sarzi-Puttini P. Fibromyalgia and sleep. Best Pract Res Clin Rheumatol 2011;25:227-39.

26. Moldofsky H, Scarisbrick P, England R, Smythe H. Musculosketal symptoms and nonREM sleep disturbance in patients with "fibrositis syndrome" and healthy subjects. Psychosom Med 1975;37:341-51.

27. Drewes AM, Nielsen KD, Taagholt SJ, Bjerregård K, Svendsen L, Gade J. Sleep intensity in fibromyalgia: focus on the microstructure of the sleep process. 
Br J Rheumatol 1995;34:629-35.

28. Pivik RT, Harman K. A reconceptualization of EEG alpha activity as an index of arousal during sleep: all alpha activity is not equal. J Sleep Res 1995;4:131-137.

29. Perlis ML, Giles DE, Bootzin RR, Dikman ZV, Fleming GM, Drummond SP, et al. Alpha sleep and information processing, perception of sleep, pain, and arousability in fibromyalgia. Int $\mathrm{J}$ Neurosci 1997;89:265-80.

30. Smith AK, Togeiro SM, Tufik S, Roizenblatt S. Disturbed sleep and musculoskeletal pain in the bed partner of patients with obstructive sleep apnea. Sleep Med 2009;10:904-12.

31. May KP, West SG, Baker MR, Everett DW. Sleep apnea in male patients with the fibromyalgia syndrome. Am J Med 1993;94:505-8.

32. Moldofsky $\mathrm{H}$, Inhaber $\mathrm{NH}$, Guinta $\mathrm{DR}$, Alvarez-Horine SB. Effects of sodium oxybate on sleep physiology and sleep/wake-related symptoms in patients with fibromyalgia syndrome: a double-blind, randomized, placebo-controlled study. J Rheumatol 2010;37:2156-66.

33. Gold AR, Dipalo F, Gold MS, Broderick J. Inspiratory airflow dynamics during sleep in women with fibromyalgia. Sleep 2004;27:459-66.

34. Prados G, Miró E, Martínez MP, Sánchez AI, López S, Sáez G. Fibromyalgia: gender differences and sleep-disordered breathing. Clin Exp Rheumatol 2013;31:102-10.

35. Cuatrecasas G, Alegre C, Casanueva FF. GH/IGF1 axis disturbances in the fibromyalgia syndrome: is there a rationale for GH treatment? Pituitary 2014;17:277-83.

36. Bennett RM. Adult growth hormone deficiency in patients with fibromyalgia. Curr Rheumatol Rep 2002;4:306-12.

37. Jones KD, Deodhar P, Lorentzen A, Bennett RM, Deodhar AA. Growth hormone perturbations in fibromyalgia: a review. Semin Arthritis Rheum 2007;36:357-79. 